\title{
Contribution of Table Banking in the Empowerment of Women Entrepreneurs in Kenya: A Case of Eldoret Town
}

\author{
Fridah Mwobobia $^{1 *}$ \\ ${ }^{1}$ Limkokwing University of Creative Technlogy, Business \& Globalization Faculty, Gaborone, \\ Botswana \\ ${ }^{*}$ Fridah Mwobobia, E-mail: 2016fridaam@gmail.com
}

\begin{abstract}
The study aimed to establish the contribution of Table banking in the empowerment of women entrepreneurs in Eldoret, Kenya. The questions explored were 1) What is Table banking, 2) Which categories of women are involved in Table banking in Eldoret, Kenya? 3) What contribution has Table banking brought in the empowerment of women entrepreneurs in Eldoret, 4) What challenges are facing women entrepreneurs as far as Table banking is concerned, 5) what can be recommended 120 women entrepreneurs from Eldoret town were randomly sampled. A questionnaire was used as the data collecting instrument. Findings revealed that Table banking has played a significant role in the empowerment of women entrepreneurs in Eldoret. Table banking has enabled women to purchase land, start and expand businesses, brought harmony at homes and financial independence.

Regarding the challenges facing women entrepreneurs, in Eldoret as far as Table banking is concerned include default cases, poor record keeping, disagreements, failure to attend meetings and failure to pay loan interests. Recommendations put forward among others are provision of training of women entrepreneurs in time and financial management, introduction of a law that would restrict women on the number of Table banking groups one joins among others. Further research in this field is recommended.
\end{abstract}

Keywords

Table banking, empowerment, women entrepreneurs, Eldoret, Kenya

\section{Introduction}

\subsection{Background to Study}

Most of the world's poor are women (Murgon \& Lumwamu, 2014). African women constitute the majority of the continents's population they however lag behind compared to men in many aspects. According to 2009 Kenyan population and housing census, women constitute 50.3 percent of Kenyans majority, majority of them have no access to mainstream banking by either choice or fate due to deep routed socio-economic and cultural factors that for a long time have worked to the women's disadvantage (Kimta, 2015). 
Poverty and illiteracy still remain the greatest challenge to Kenyan women especially the rural folk. Poverty level among Kenyan women currently stands at 25 percent (Limobk, 2014). Tubey (2013) recommended that women entrepreneurs need to be supported because they bring fresh motivation to the business sector.

In order to assist the unbanked women in Kenya, Table banking was initially developed by the Poverty Eradication Commission (PEC) under the former Ministry of Planning and Vision 2030, targeting Millennium Development Goals 1 eradicating abject poverty especially in the rural areas in Kenya (softkenya.com). Mrs. Rachel Ruto - the wife to the kenyan Deputy President emphasizes that PEC was created with the aim to help women in the low income chamas to access finances to start income generating projects. Table banking's objective is to bring financial services to the poor, particularly the poorest women - to help them fight poverty, stay profitable and financially sound (ROK, 2009). Table banking follows the same concept as merry-go-round but, for the former money contributed by members is given to members as loans which they pay back in the following meeting with an interest (Njuguna, 2015). It operates as a Savings and Credit Cooperative Society (SACCO), where members save and borrow from their collection for short term or long term at a low rate interest (Kaino, 2013). Its an intervention for the poor (Kariuki \& Ngugi, 2014). Another objective of Table banking is to give women economic empowerment through financial assistance and trainings (Murithi, 2014).

Today, in Kenya Mrs Ruto is facilitating Table banking through Joyful Women Organization (JOYWO), which was started in 2009 in Uasin Gishu (Eldoret North) with the aim of empowering women farmers (Gitau, 2011). The objective was to safeguard the welfare of women in the fight against poverty (Kaino, 2013). This is a concept that encourages women to boost their savings and investments (Isiye \& Masava, 2013). Table banking program has been launched in 37 counties: Uasin Gishu, Nandi, Nairobi, TransNzoia, Bomet, Kericho among others, benefitting more than 170,000 women who are revolving $\quad$ about $\quad$ Kes 1.2 billion $\quad$ by $\quad$ November 2014 (http://www.uhuru-encourages-women-on-table-banking). According to Limo (2014) once the Table banking group has been launched it operates under the umbrella of JOYWO, an Non Governmental Organization that enables them to enjoy capacity building, financial donations and grants from donors. JOYWO was formed to empower Kenyan women economically and enhance their house-hold food security by engaging in savings and credit scheme-Table banking. JOYWO seeks to achieve variety of objectives among them: 
- Enhancing access to financial resources;

- Supporting investment growth;

- $\quad$ Strengthening livelihood projects (http://www.joywo.org/about-us/).

The Executive Director of JOYWO says that organization main aim is to rescue women from poverty because women are hardworking and have continuously contributed to the national development of the country (Nyaga, 2013). In addition Too (2013) states that "Mrs Ruto added that JOYWO can assist women groups make business proposals and business plans empowering them economically and reducing poverty especially in rural areas”.

The model encourages women to form a group of twenty five members or below and bring their income on the table and distribute to members in form of loans, according to by-laws that guide borrowing, lending and recovery practices (Limo, 2014). In addition Kariuki et al. (2014) describes Table banking as a group based funding strategy from which members save and borrow instantly. It is an empowerment programme that cares for micro and small business owners who need credit to finance their businesses but cannot qualify from formal banks due to factors such as lack of collateral, long distances, high interest rates (Kariuki et al., 2014; ROK, 2009). With Table banking, a women group can allow long-term borrowers to repay at 1 percent interest rate over 3 years or 36 months. A short-term borrower to pay at 10 percent interest within 30 days. Table banking is purely based on mutual trust, openness and honesty for the fellow members to guarantee each other's savings. To cater for defaulters, members who could have problem with repayment are requested to notify the group on time to either be assisted or have their repayment period extended. In cases where the member fails to pay and does not notify members, a committee is formed to investigate. Later if nothing is done members are forced to take the defaulters household goods and report the member to the area chief (Obiria, 2015).

Table banking adopts a model of Grameen bank in Bangladesh and the village savings \& loans schemes of Zanzibar. Grameen bank is one of the successful credit banks, founded by Dr Yunus an economist, who pursued the philosophy that "the less one has the more attractive one is" the opposite of the conventional banks policies. Grameen Bank gave and gives priority to the poor, rootless, landless and vulnerable with 97 percent borrowers being women. Kariuki and Ngugi (2014) emphasize that Grameen Bank borrowers are women who constitute the weakest group among the poor in rural areas. It provides micro-loans without requiring any collateral or mortgage. The objective of the Grameen 
Bank is to fight against poverty, by proving loans to start Small Micro Enterprises or any other business to turn out from poverty. Its aim is to replace "hands of beggar into the hands of the worker" (Rahman \& Khaled, 2011)

Ruto urges women to join hands in pooling resources to help overcome poverty and illiteracy especially among the rural women (African Business Forum, 2014). Following the success of Table banking the Kenyan Government has introduced Uwezo Fund which offers more monetary resources to women, youth and persons living with disability (http://www.uwezo.go.ke/).

\subsection{Statement of the Problem}

The research aimed to investigate the contribution of Table banking towards empowering of women entrepreneurs in Eldoret, Kenya. Table banking is a strategy of self help organization that offers unsecured loans to women - a way to access loans without going through a microfinance organization (African Business Forum, 2014). The study was based in Eldoret, the largest city and administrative capital of Uasin Gishu county. Eldoret is among the fastest growing cities in Kenya. Its main economic activities are maize farming, Dairy farming, horticulture, sports tourism; manufacturing sector which is dominated by textile and wood factories. Eldoret is the fifth largest city in Kenya with three constituencies: Eldoret North, Eldoret South and Eldoret East. Between 2005-2006 poverty rate in Uasin Gishu was 44.5 percent (Municipal Council of Eldoret, 2011). Eldoret has a population of 280,000 people.

Table banking program has been launched in 37 counties among them is Uasin Gishu where Eldoret is situated. According to Kaino (2013), Table banking was started to help women access money to engage in income generating activities in Mrs Rachel Ruto's home-Uasin Gishu. Has it met the objective? According to Johncraig (2012), Table banking in Eldoret has enabled women to get involved in much income generating projects such as green houses, rearing milk goats among others which enables them to get investment returns of over 200 percent. Is this a fact?

Table banking groups are slowly becoming the main source of small loans for women in East Africa (Mengo, 2014). The main objective of Table banking is to pool resources and then lend to the members who are in need.

Quail and Flood (2014) reported that extreme poverty in Eldoret drives men, women and children in the rubbish dump to look for valuables that can be sold as a means of livelihood making $£ 1.30$ per day. Many women have positive testimonies about Table banking: women from Bandiat a women group in 
Kapseret claim there is women prosperity, provision for families, access to low interest loans, have received dividends, have engaged horticulture, able to educate their children and have received training in passion fruits farming among others (Kaino, 2013). In addition a Table banking beneficiary from Busia County, confesses that it has enabled her provide for her family, meets her child's school requirements and has generally brought peace in the community (Ochieng, 2011). Mengo (2014) reports that Table banking has enabled women to avoid lengthy procedures of obtaining loans and also cut them off from expensive loans with interest rates as high as 26 percent, It has become the main source of small loans for women in East Africa. Is this inclusive of Eldoret women?

Table banking has become a great beneficiary to many women in different counties who have started many activities such as: dry-land fish farming, rabbit farming poultry keeping even to the disabled woman (http//www.Action.actionaid.org). In addition Obiria (2015) stresses that Table banking has boosted many women social and economic standards, women no longer depend on husbands for money and it has reduced quarrels over money. Are such benefits being enjoyed by women in Eldoret? Mosop is a women group in Rongai that wishes to save and get loans through Table banking and break away from the cycle of poverty. This group has 25 members and each of them has a success story to tell. Many women from this group have claimed success from the association ranging from ability to pay University fees for their children to starting businesses, engaging in agriculture, gaining financial stability, buying land and provision to the family (Obiria, 2014). What about in Eldoret which is among the first county where Table banking was launched? The same group gets empowered with entrepreneurial skills by Rongai Social Economic Women Organization (ROSEWO) an NGO contracted by Devolution Ministry. Is such happening in Eldoret?

In the backdrop of this, the study sought to find out how Table banking has positively contributed to the empowerment of women entrepreneurs in Eldoret.

\subsubsection{Objectives of the Study}

Specifically, the objectives of the study were:

1) To examine the concept Table banking;

2) To explore the categories of women entrepreneurs involved in Table banking in Eldoret;

3) To examine the contribution Table banking has brought towards the empowerment of women entrepreneurs in Eldoret;

4) To identify the challenges women entrepreneurs face in Eldoret in the process of Table banking; 
5) To recommend the way forward as far as Table banking and women entrepreneurs is concerned.

\subsubsection{Research Questions}

1) What is Table banking?

2) Which categories of women entrepreneurs are involved in Table banking in Eldoret?

3) What contribution has Table banking brought towards empowerment of women entrepreneurs in Eldoret?

4) What challenges are women entrepreneurs facing as far as Table banking is concerned?

5) What can be recommended for future as far as Table banking is concerned?

\subsection{Significance of Study}

The study established the contribution Table banking has brought towards the empowerment of women entrepreneurs in Eldoret and identified the challenges women face as far as Table banking is concerned. Lessons learnt here could be used to highlight areas of improvement for Table banking groups in Eldoret and other areas. The study is a humble contribution to the body of knowledge regarding Savings \& Credit and women entrepreneurs in Kenya and other developing countries.

\section{Method}

\subsection{Research Design}

The study adopted a combined research approach of both positivism (quantitative) and phenomenological (qualitative) paradigms. This was due to the nature of data collected and it also increased reliability and validity of the findings. The use of combined design allowed the researcher to identify the relationships between variables and analyzed concrete cases. This approach is balanced and helped the researcher balance the weaknesses or blind spots of the other design (Gray, 2009). Data collected involved women entrepreneurs and Table banking groups.

\subsection{Population and Sample}

The population comprised of women entrepreneurs based in Eldoret town, who were randomly sampled and 120 women entrepreneurs were selected.

\subsection{Instrumentation}

A structured questionnaire was compiled for data collection. It was carefully worded to avoid offending the respondents and was divided into three sections following the variables in the study. It consisted closed and open ended questions. For the closed ended questions five likert scales was used. Before 
administration of the questionnaire a pre-test was done to test its validity and reliability Corrections were done in order to maximize returns and minimize the error rate. Questionnaire was used because it is cheaper in terms of time and money and provides quick inflow of data (Gray, 2009).

\subsection{Data Collection}

Sample survey was employed in this study. Three research assistants were hired and trained on how to administer the research questionnaires. Data was collected between the months of June-July 2015.

\subsection{Data Analysis}

With the research design having adopted a mixed approach, the analysis followed suit and adopted both quantitative and qualitative analysis. The Statistical Package for Social Sciences (SPSS) version 21 was used to analyze the collected data. To establish the relationship between independent variables and dependent variables correlation analysis was used. For qualitative, descriptive and inferential methods of data analysis were used. Regression analysis was used to establish the relationship among predictors and dependent variables.

\section{Results}

Table 1. Respondents Age

\begin{tabular}{|c|c|c|c|c|c|}
\hline & & Frequency & Percent & Valid Percent & Cumulative Percent \\
\hline \multirow{5}{*}{ Valid } & $10-19$ & 4 & 5.0 & 5.0 & 5.0 \\
\hline & $20-29$ & 33 & 41.3 & 41.3 & 46.3 \\
\hline & $30-39$ & 25 & 31.3 & 31.3 & 77.5 \\
\hline & 40 and above & 18 & 22.5 & 22.5 & 100.0 \\
\hline & Total & 80 & 100.0 & 100.0 & \\
\hline
\end{tabular}

Table 2. Marital Status of the Respondents

\begin{tabular}{|c|c|c|c|c|c|}
\hline & & Frequency & Percent & t Valid Percent & Cumulative Percent \\
\hline \multirow{3}{*}{ Valid } & Married & 29 & 36.3 & 36.3 & 36.3 \\
\hline & Single & 48 & 60.0 & 60.0 & 96.3 \\
\hline & \multicolumn{2}{|c|}{ Divorced3 } & 3.8 & 3.8 & 100.0 \\
\hline
\end{tabular}




\begin{tabular}{llll}
\hline Total & 80 & 100.0 & 100.0 \\
\hline
\end{tabular}

Table 3. Respondents Level of Education

\begin{tabular}{|c|c|c|c|c|c|}
\hline & & Frequency & Percent & Valid Percent & Cumulative Percent \\
\hline \multirow{7}{*}{ Valid } & primary & 10 & 12.5 & 12.5 & 12.5 \\
\hline & secondary & 21 & 26.3 & 26.3 & 38.8 \\
\hline & Tertiary & 45 & 56.3 & 56.3 & 95.0 \\
\hline & 4.00 & 1 & 1.3 & 1.3 & 96.3 \\
\hline & 6.00 & 1 & 1.3 & 1.3 & 97.5 \\
\hline & 7.00 & 2 & 2.5 & 2.5 & 100.0 \\
\hline & Total & 80 & 100.0 & 100.0 & \\
\hline
\end{tabular}

Table 4. Industry That Respondents Belong to

\begin{tabular}{|c|c|c|c|c|c|}
\hline & & Frequency & Percent & Valid Percent & Cumulative Percent \\
\hline & farming & 11 & 13.8 & 13.9 & 13.9 \\
\hline & \multicolumn{2}{|c|}{ Hair \& Beauty 26} & 32.5 & 32.9 & 46.8 \\
\hline & Finance & 22 & 27.5 & 27.8 & 74.7 \\
\hline & \multicolumn{2}{|c|}{ Manufacturing 5} & 6.3 & 6.3 & 81.0 \\
\hline \multirow[t]{5}{*}{ Valid } & Tourism & 6 & 7.5 & 7.6 & 88.6 \\
\hline & Textile & 3 & 3.8 & 3.8 & 92.4 \\
\hline & Others & 4 & 5.0 & 5.1 & 97.5 \\
\hline & 8.00 & 2 & 2.5 & 2.5 & 100.0 \\
\hline & Total & 79 & 98.8 & 100.0 & \\
\hline \multicolumn{2}{|c|}{ MissingSystem } & 1 & 1.3 & & \\
\hline \multicolumn{2}{|l|}{ Total } & 80 & 100.0 & & \\
\hline
\end{tabular}

Table 5. Respondents Length of Experience of in Business

\begin{tabular}{llllll}
\hline & Frequency & Percent & Valid Percent & Cumulative Percent \\
\hline Valid & less than a year & 22 & 27.5 & 27.5 & 27.5 \\
\hline
\end{tabular}




\begin{tabular}{lllll}
\hline 1 year & 18 & 22.5 & 22.5 & 50.0 \\
2-3 years & 20 & 25.0 & 25.0 & 75.0 \\
4-5 years & 9 & 11.3 & 11.3 & 86.3 \\
Above 5 years & 10 & 12.5 & 12.5 & 98.8 \\
6.00 & 1 & 1.3 & 1.3 & 100.0 \\
Total & 80 & 100.0 & 100.0 & \\
\hline
\end{tabular}

Table 6. Contribution of Table Banking towards Women Empowerment in Eldoret

\begin{tabular}{|c|c|c|c|c|c|c|c|c|c|c|c|c|}
\hline & $\mathrm{N}$ & & Mean & Std. & Variance & Skewness & Std. Error of & Kurtosis & Std. Error & Range & Minimum & Maximum \\
\hline & Valid & Missing & & Deviation & & & Skewness & & of Kurtosis & & & \\
\hline startbusiness & 80 & 0 & 1.3375 & .57244 & .328 & 1.503 & .269 & 1.329 & .532 & 2.00 & 1.00 & 3.00 \\
\hline Expandbusiness & 80 & 0 & 1.4625 & .77857 & .606 & 1.778 & .269 & 2.692 & .532 & 3.00 & 1.00 & 4.00 \\
\hline Buyland & 79 & 1 & 1.7468 & 1.00599 & 1.012 & 1.541 & .271 & 1.992 & .535 & 4.00 & 1.00 & 5.00 \\
\hline ContributehomeBudgets & 80 & 0 & 1.7125 & .98333 & .967 & 1.513 & .269 & 2.024 & .532 & 4.00 & 1.00 & 5.00 \\
\hline BroughtHarmonytohomes & 80 & 0 & 1.8875 & 2.41825 & 5.848 & 7.494 & .269 & 62.295 & .532 & 21.00 & 1.00 & 22.00 \\
\hline Finindependence & 80 & 0 & 1.6250 & 94635 & .896 & 1.743 & .269 & 3.003 & .532 & 4.00 & 1.00 & 5.00 \\
\hline Loansaccessible & 80 & 0 & 1.5375 & .85601 & .733 & 1.930 & .269 & 3.942 & .532 & 4.00 & 1.00 & 5.00 \\
\hline HelpedDisabledcomm & 80 & 0 & 1.7500 & .89301 & .797 & 1.613 & .269 & 3.337 & .532 & 4.00 & 1.00 & 5.00 \\
\hline Womentraining & 80 & 0 & 1.6500 & .85832 & .737 & 1.489 & .269 & 2.458 & .532 & 4.00 & 1.00 & 5.00 \\
\hline Foodsecurity & 80 & 0 & 2.0000 & 1.06735 & 1.139 & 1.153 & .269 & 1.113 & .532 & 4.00 & 1.00 & 5.00 \\
\hline Wreformed & 80 & 0 & 1.9250 & 1.13377 & 1.285 & 1.166 & .269 & .700 & .532 & 4.00 & 1.00 & 5.00 \\
\hline wbuildhouses & 80 & 0 & 1.7000 & .68251 & .466 & .951 & .269 & 1.629 & .532 & 3.00 & 1.00 & 4.00 \\
\hline Wlivingstds & 80 & 0 & 1.7125 & 90279 & .815 & 1.666 & .269 & 3.361 & .532 & 4.00 & 1.00 & 5.00 \\
\hline Wdairypoultry & 80 & 0 & 1.5500 & .67317 & .453 & .835 & .269 & -.421 & .532 & 2.00 & 1.00 & 3.00 \\
\hline Wdividends & 80 & 0 & 1.7250 & .84156 & .708 & 1.216 & .269 & 1.826 & .532 & 4.00 & 1.00 & 5.00 \\
\hline
\end{tabular}




\begin{tabular}{|c|c|c|c|c|c|c|c|c|c|c|c|c|}
\hline Whorticulture & 80 & 0 & 1.7875 & 1.44690 & 2.094 & 4.857 & .269 & 31.606 & .532 & 11.00 & 1.00 & 12.00 \\
\hline Wmarket & 80 & 0 & 1.5875 & .85231 & .726 & 1.796 & .269 & 3.596 & .532 & 4.00 & 1.00 & 5.00 \\
\hline Wmodernfarming & 79 & 1 & 1.8861 & 1.41410 & 2.000 & 4.923 & .271 & 33.498 & .535 & 11.00 & 1.00 & 12.00 \\
\hline
\end{tabular}

Table 7. Challenges Facing Women Entrepreneurs as Far as Table Banking is Concerned in

\section{Eldoret}

\begin{tabular}{|c|c|c|c|c|c|c|}
\hline & wdefaultloans & nonpaymentswdisagreements & Defaultcasesintodeath & Wmissmonthlymeetings & Recordkepingprbm & Wfailpaymonthlyinterest \\
\hline Valid & 80 & 80 & 80 & 80 & 80 & 80 \\
\hline Missing & 0 & 0 & 0 & 0 & 0 & 0 \\
\hline Mean & 1.7375 & 1.8125 & 2.4750 & 1.8625 & 2.3375 & 1.8125 \\
\hline Median & 1.0000 & 2.0000 & 2.0000 & 2.0000 & 2.0000 & 2.0000 \\
\hline Mode & 1.00 & 1.00 & $1.00^{\mathrm{a}}$ & 2.00 & 2.00 & 1.00 \\
\hline Std. Deviation & .95126 & .94258 & 1.39597 & .82283 & 1.23189 & .94258 \\
\hline Variance & .905 & .888 & 1.949 & .677 & 1.518 & .888 \\
\hline Skewness & 1.278 & 1.225 & .697 & 1.102 & .907 & 1.411 \\
\hline Std. Error of Skewness & .269 & .269 & .269 & .269 & .269 & .269 \\
\hline Kurtosis & 1.153 & 1.197 & -.722 & 1.974 & -.047 & 2.183 \\
\hline Std. Error of Kurtosis & .532 & .532 & .532 & .532 & .532 & .532 \\
\hline Range & 4.00 & 4.00 & 4.00 & 4.00 & 4.00 & 4.00 \\
\hline
\end{tabular}

Table 8. Strategies Put Forward to Improve Table banking among Women Entrepreneurs

\begin{tabular}{lllll} 
& Collateralintroduced & Weducatedfinmgmt & Weducatedtimemgmt & Restrictionsontbgrps \\
\hline $\mathrm{N}$ & 80 & 80 & 80 & 80 \\
\hline
\end{tabular}




\begin{tabular}{|c|c|c|c|c|c|}
\hline & Missing & 0 & 0 & 0 & 0 \\
\hline Mean & & 1.8000 & 1.5125 & 1.4250 & 1.9500 \\
\hline Median & & 2.0000 & 1.0000 & 1.0000 & 2.0000 \\
\hline Mode & & 1.00 & 1.00 & 1.00 & 1.00 \\
\hline Std. Deviation & & .94668 & .77938 & .67082 & 1.16814 \\
\hline Variance & & .896 & .607 & .450 & 1.365 \\
\hline Skewness & & 1.241 & 2.097 & 2.345 & 1.223 \\
\hline Std. Error of Skewness & & .269 & .269 & .269 & .269 \\
\hline Kurtosis & & 1.198 & 5.734 & 9.033 & .612 \\
\hline Std. Error of Kurtosis & & .532 & .532 & .532 & .532 \\
\hline Range & & 4.00 & 4.00 & 4.00 & 4.00 \\
\hline Minimum & & 1.00 & 1.00 & 1.00 & 1.00 \\
\hline
\end{tabular}

Table 9. Correlation Analysis

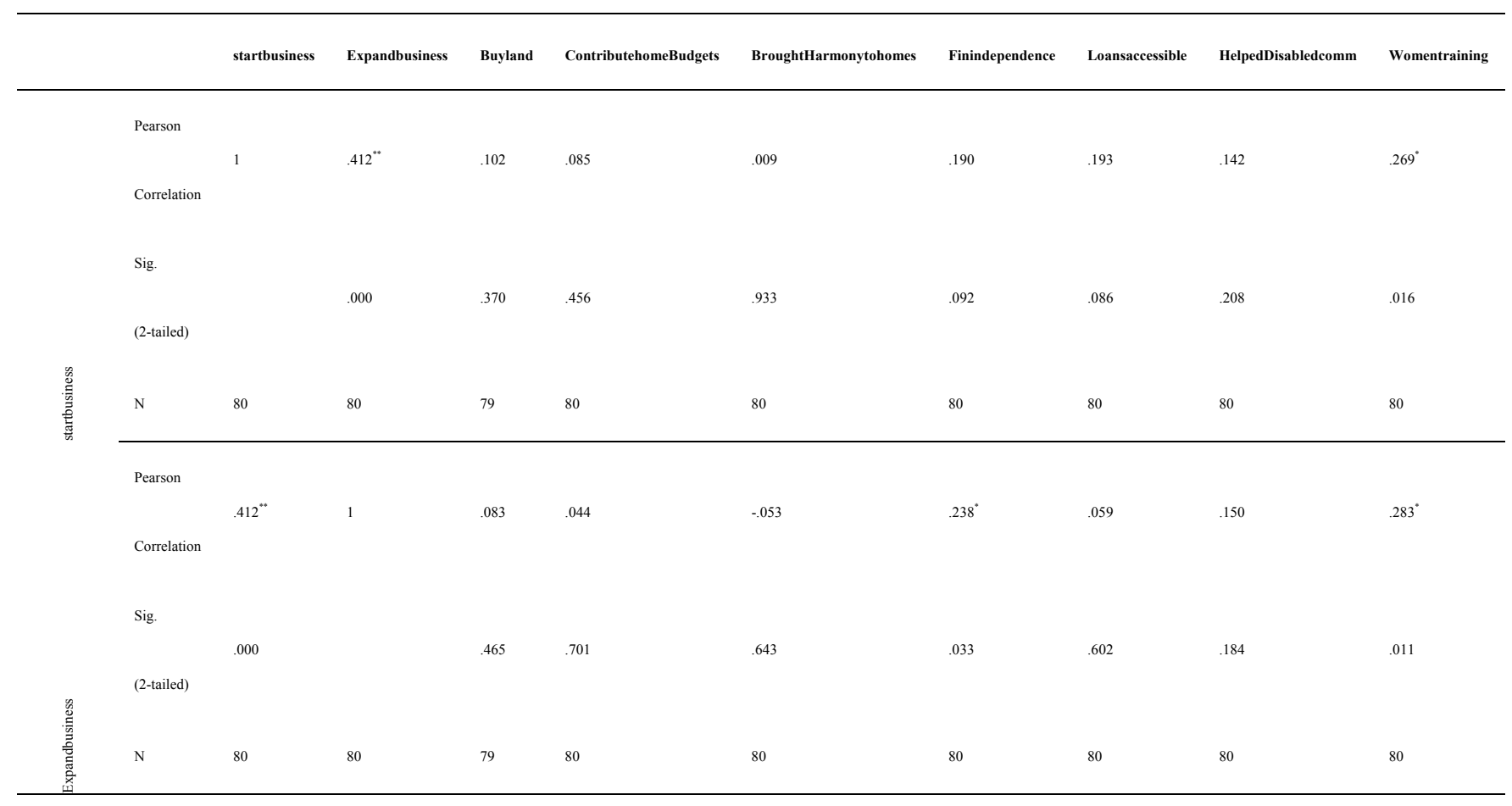




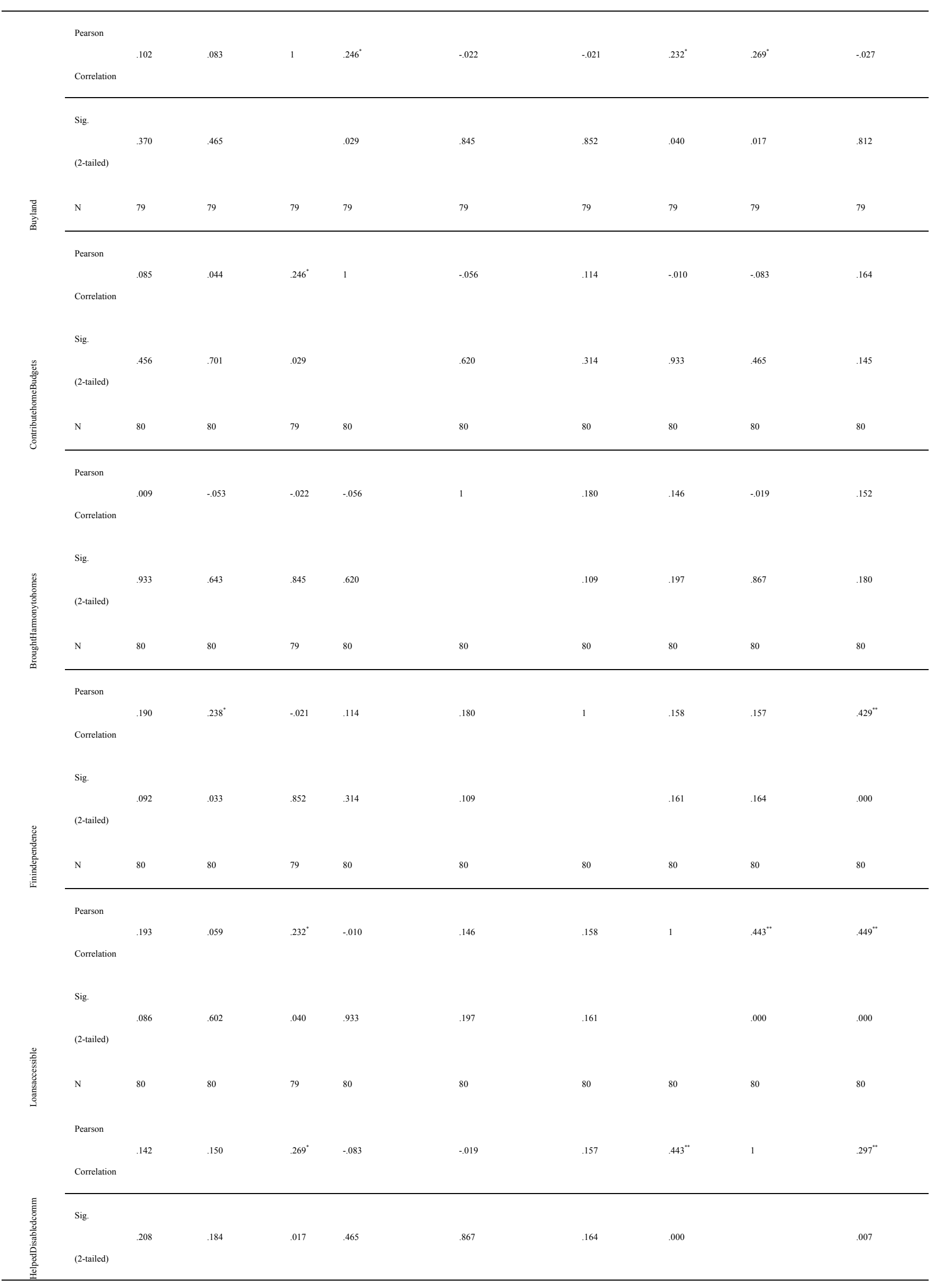




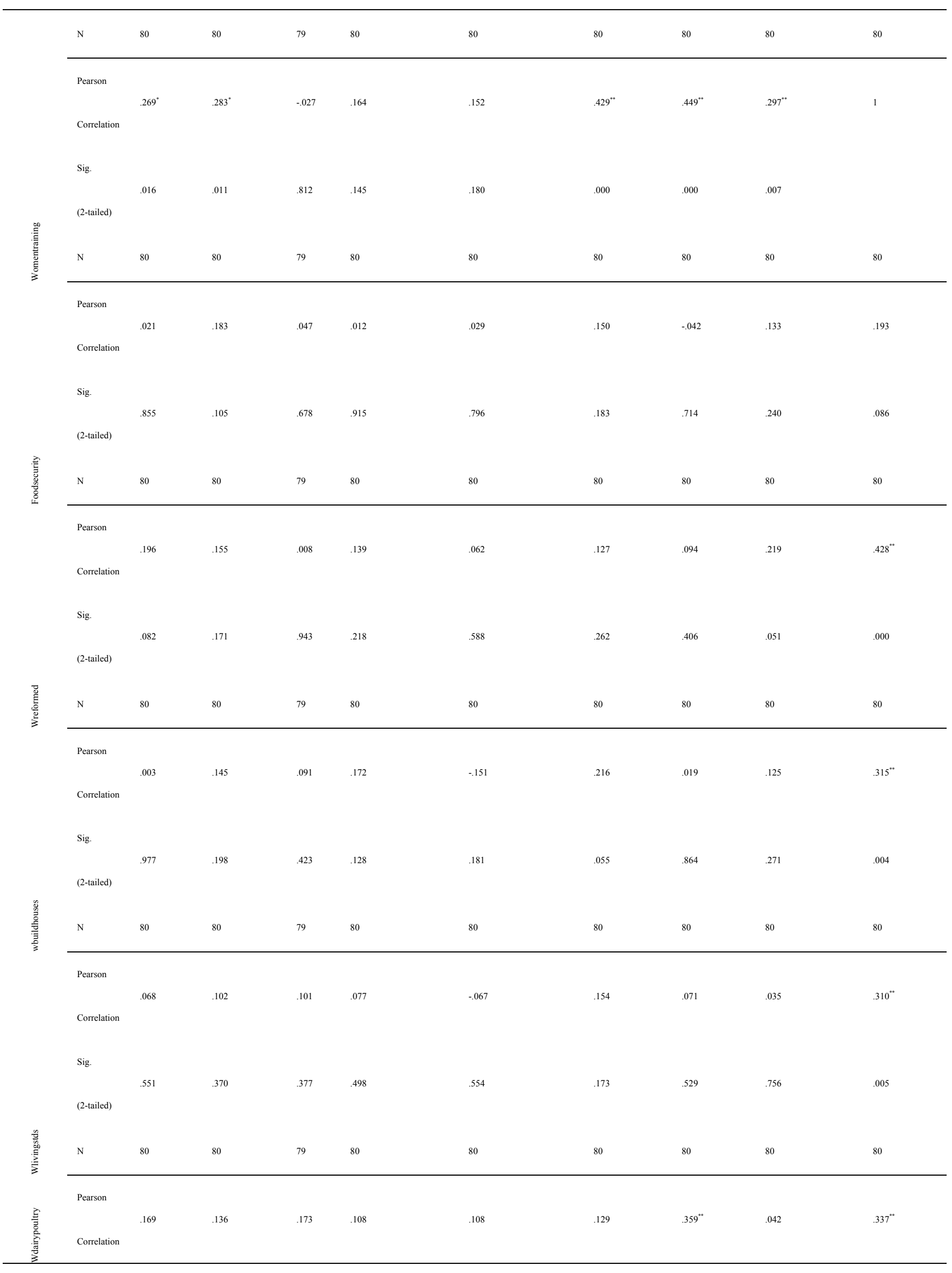




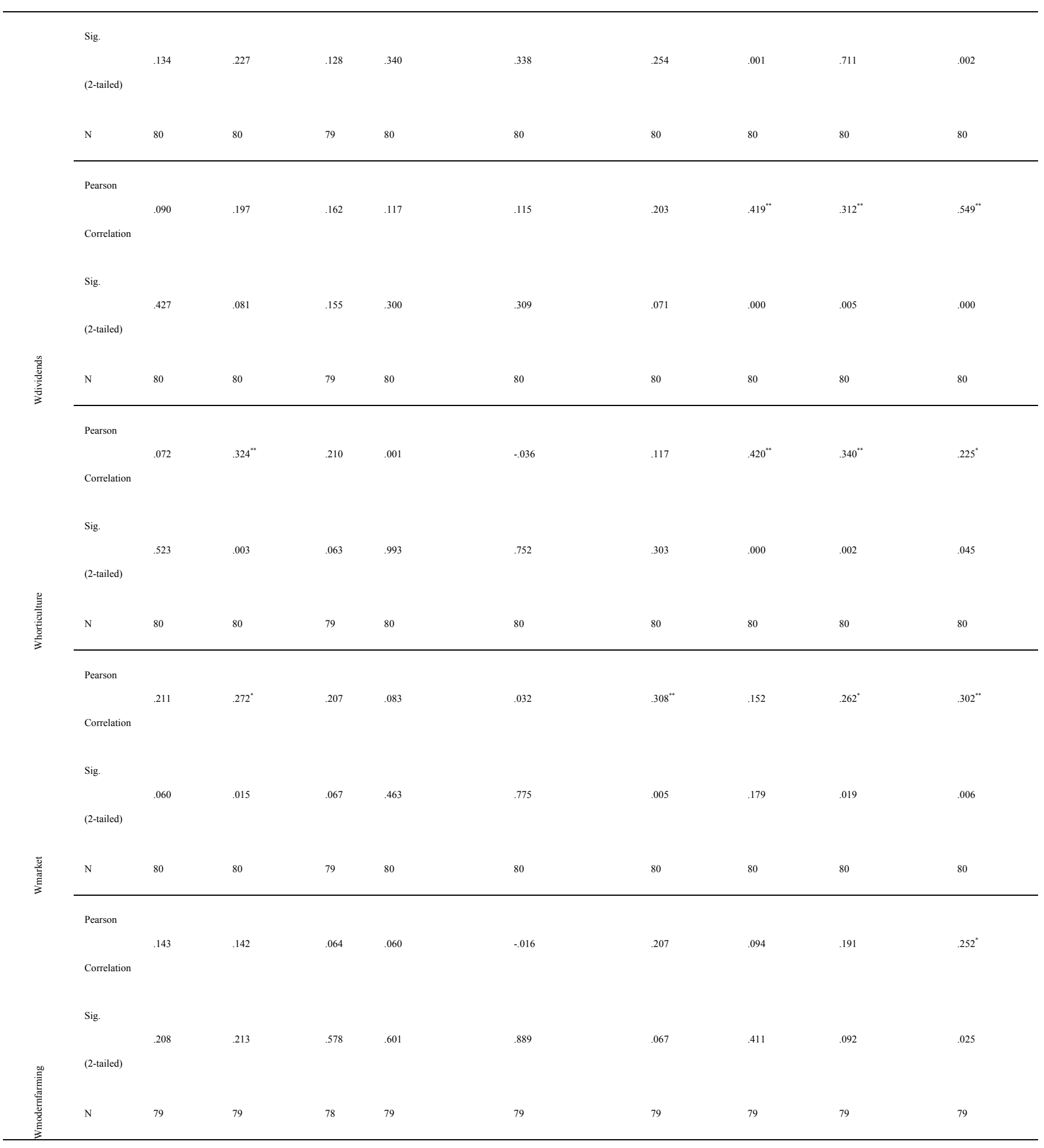

\begin{tabular}{|c|c|c|c|c|c|c|c|c|c|c|}
\hline & & Foodsecurity & Wreformed & wbuildhouses & Wlivingstds & Wdairypoultry & Wdividends & Whorticulture & Wmarket & Wmodernfarming \\
\hline & Pearson & .021 & .196 & .003 & .068 & .169 & .090 & .072 & .211 & .143 \\
\hline & Sig. (2-tailed) & .855 & .082 & .977 & .551 & .134 & .427 & .523 & .060 & .208 \\
\hline 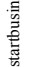 & $\mathrm{N}$ & 80 & 80 & 80 & 80 & 80 & 80 & 80 & 80 & 79 \\
\hline
\end{tabular}




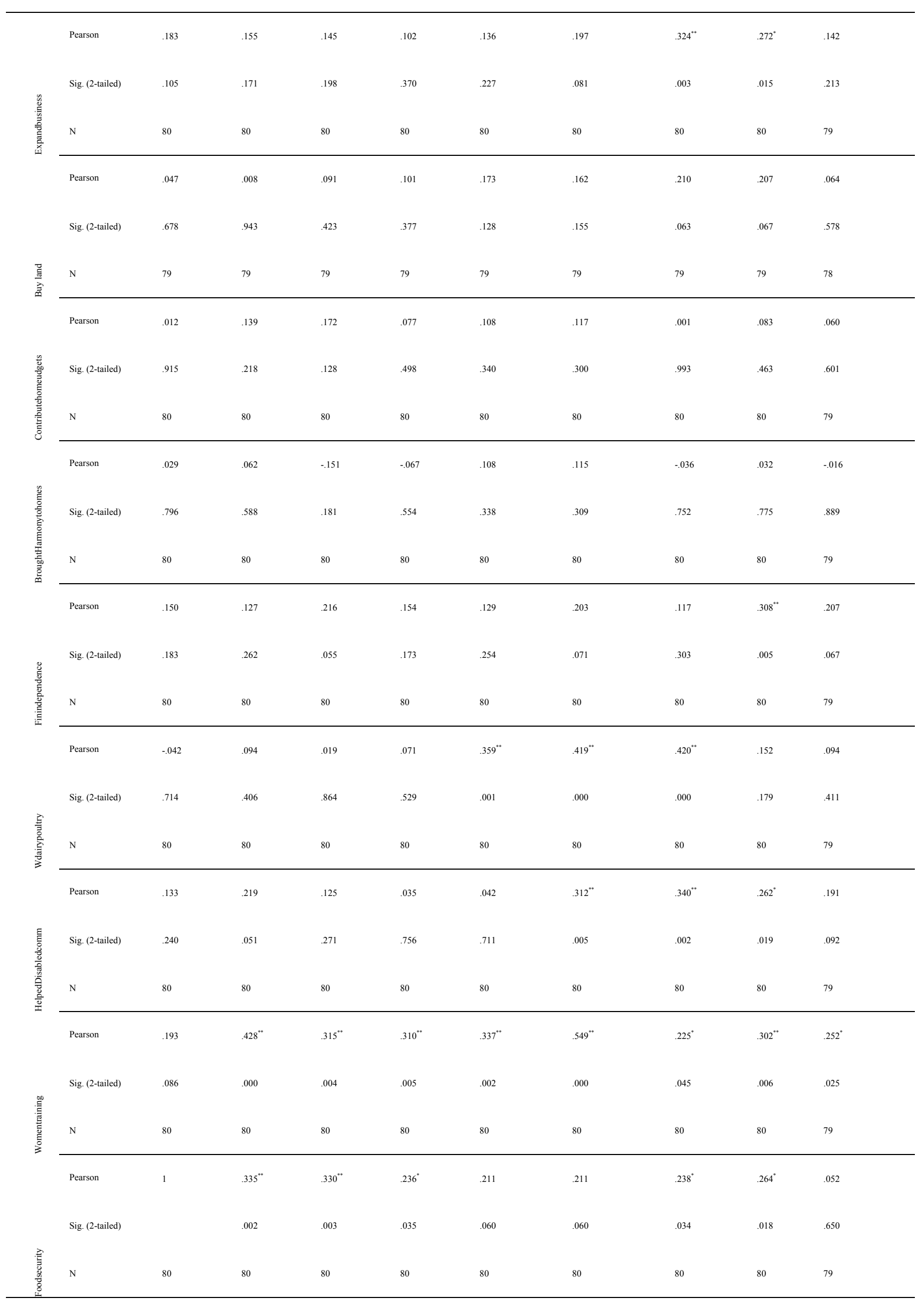




\begin{tabular}{|c|c|c|c|c|c|c|c|c|c|c|}
\hline & Pearson & $.335^{* \prime}$ & 1 & $.330^{\circ}$ & $.313^{*}$ & .071 & .137 & .144 & .190 & $.253^{\circ}$ \\
\hline \multirow{3}{*}{ 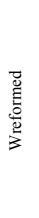 } & Sig. (2-tailed) & .002 & & .003 & .005 & .530 & .225 & .201 & .091 & .025 \\
\hline & $\mathrm{N}$ & 80 & 80 & 80 & 80 & 80 & 80 & 80 & 80 & 79 \\
\hline & Pearson & $.330^{\circ *}$ & $.330^{* *}$ & 1 & $.372^{* \prime}$ & .116 & .185 & $.229^{\circ}$ & $.242^{\circ}$ & $.258^{\circ}$ \\
\hline \multirow{3}{*}{ 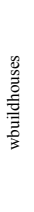 } & Sig. (2-tailed) & .003 & .003 & & .001 & .307 & .100 & .041 & .031 & .022 \\
\hline & $\mathrm{N}$ & 80 & 80 & 80 & 80 & 80 & 80 & 80 & 80 & 79 \\
\hline & Pearson & $.236^{\circ}$ & $.313^{*}$ & $.372^{* *}$ & 1 & $.263^{\circ}$ & .195 & .098 & .058 & .055 \\
\hline \multirow{3}{*}{ 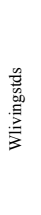 } & Sig. (2-tailed) & .035 & .005 & .001 & & .018 & .084 & .387 & .611 & .630 \\
\hline & $\mathrm{N}$ & 80 & 80 & 80 & 80 & 80 & 80 & 80 & 80 & 79 \\
\hline & Pearson & .211 & .071 & .116 & $.263^{\circ}$ & 1 & $.606^{*}$ & $.407^{* \circ}$ & .202 & .054 \\
\hline \multirow{2}{*}{ 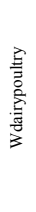 } & Sig. (2-tailed) & .060 & .530 & .307 & .018 & & .000 & .000 & .073 & .637 \\
\hline & Pearson & .211 & .137 & .185 & .195 & $.606^{*}$ & 1 & $.367^{* *}$ & $.316^{\circ "}$ & $.243^{\circ}$ \\
\hline \multirow{3}{*}{ 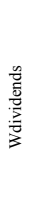 } & Sig. (2-tailed) & .060 & .225 & .100 & .084 & .000 & & .001 & .004 & .031 \\
\hline & $\mathrm{N}$ & 80 & 80 & 80 & 80 & 80 & 80 & 80 & 80 & 79 \\
\hline & Pearson & $.238^{\circ}$ & .144 & $.229^{\circ}$ & .098 & $.407^{* *}$ & $.367^{* *}$ & 1 & $.226^{\circ}$ & $.263^{\circ}$ \\
\hline \multirow{2}{*}{ 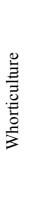 } & Sig. (2-tailed) & .034 & .201 & .041 & .387 & .000 & .001 & & .044 & .019 \\
\hline & Pearson & $.264^{\circ}$ & .190 & $.242^{\circ}$ & .058 & .202 & $.316^{*}$ & $.226^{\circ}$ & 1 & $.258^{\circ}$ \\
\hline \multirow{3}{*}{ 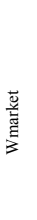 } & Sig. (2-tailed) & .018 & .091 & .031 & .611 & .073 & .004 & .044 & & .022 \\
\hline & $\mathrm{N}$ & 80 & 80 & 80 & 80 & 80 & 80 & 80 & 80 & 79 \\
\hline & Pearson & .052 & $.253^{\circ}$ & $.258^{\circ}$ & .055 & .054 & $.243^{\circ}$ & $.263^{\circ}$ & $.258^{\circ}$ & 1 \\
\hline \multirow{2}{*}{ 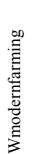 } & Sig. (2-tailed) & .650 & .025 & .022 & .630 & .637 & .031 & .019 & .022 & \\
\hline & $\mathrm{N}$ & 79 & 79 & 79 & 79 & 79 & 79 & 79 & 79 & 79 \\
\hline
\end{tabular}

** Correlation is significant at the 0.01 level (2-tailed).

* Correlation is significant at the 0.05 level (2-tailed). 
Table 10. Model Summary ${ }^{k}$

\begin{tabular}{|c|c|c|c|c|c|c|c|c|c|c|}
\hline Model & $\mathrm{R}$ & R Square & Adjusted R Square & Std. Error of the Estimate & Change Statistics & & & & & Durbin-Watson \\
\hline & & & & & R Square Change & F Change & df1 & df2 & Sig. F Change & \\
\hline 1 & $.644^{\mathrm{a}}$ & .414 & .406 & 3.77140 & .414 & 53.736 & 1 & 76 & .000 & \\
\hline 2 & $.785^{\mathrm{b}}$ & .616 & .605 & 3.07503 & .201 & 39.319 & 1 & 75 & .000 & \\
\hline 3 & $.867^{\circ}$ & .752 & .741 & 2.48916 & .136 & 40.460 & 1 & 74 & .000 & \\
\hline 4 & $.928^{\mathrm{d}}$ & .860 & .853 & 1.87884 & .109 & 56.885 & 1 & 73 & .000 & \\
\hline 5 & $.952^{\circ}$ & .906 & .899 & 1.55600 & .045 & 34.435 & 1 & 72 & .000 & \\
\hline 6 & $.965^{\mathrm{f}}$ & .931 & .925 & 1.34270 & .025 & 25.693 & 1 & 71 & .000 & \\
\hline 7 & $.979^{\mathrm{g}}$ & .959 & .955 & 1.03692 & .029 & 49.048 & 1 & 70 & .000 & \\
\hline 8 & $.989^{\mathrm{h}}$ & .978 & .975 & .77487 & .018 & 56.352 & 1 & 69 & .000 & \\
\hline 9 & $.995^{i}$ & .989 & .988 & .53672 & .012 & 75.819 & 1 & 68 & .000 & \\
\hline 10 & $1.000^{\mathrm{j}}$ & 1.000 & 1.000 & . 00000 & . 011 & \multicolumn{2}{|c|}{640617776431} & 67 & .000 & 1.778 \\
\hline
\end{tabular}

a. Predictors: (Constant), Womentraining;

b. Predictors: (Constant), Womentraining, BroughtHarmonytohomes;

c. Predictors: (Constant), Womentraining, BroughtHarmonytohomes, Wmodernfarming;

d. Predictors: (Constant), Womentraining, BroughtHarmonytohomes, Wmodernfarming, Buyland;

e. Predictors: (Constant), Womentraining, BroughtHarmonytohomes, Wmodernfarming, Buyland,

Finindependence;

f. Predictors: (Constant), Womentraining, BroughtHarmonytohomes, Wmodernfarming, Buyland,

Finindependence, Expandbusiness;

g. Predictors: (Constant), Womentraining, BroughtHarmonytohomes, Wmodernfarming, Buyland, Finindependence, Expandbusiness, ContributehomeBudgets; 
h. Predictors: (Constant), Womentraining, BroughtHarmonytohomes, Wmodernfarming, Buyland, Finindependence, Expandbusiness, ContributehomeBudgets, Loansaccessible;

i. Predictors: (Constant), Womentraining, BroughtHarmonytohomes, Wmodernfarming, Buyland, Finindependence, Expandbusiness, ContributehomeBudgets, Loansaccessible, wbuildhouses;

j. Predictors: (Constant), Womentraining, BroughtHarmonytohomes, Wmodernfarming, Buyland, Finindependence, Expandbusiness, ContributehomeBudgets, Loansaccessible, wbuildhouses, startbusiness;

k. Dependent Variable: wempo.

Table 11. ANOVA ${ }^{\mathrm{a}}$

\begin{tabular}{|c|c|c|c|c|c|c|}
\hline \multicolumn{2}{|c|}{ Model } & \multirow{2}{*}{$\begin{array}{l}\text { Sum of Squares } \\
764.310\end{array}$} & \multirow{2}{*}{$\begin{array}{l}\mathbf{d f} \\
1\end{array}$} & \multirow{2}{*}{$\begin{array}{l}\text { Mean Square } \\
764.310\end{array}$} & \multirow{2}{*}{$\begin{array}{l}\mathbf{F} \\
53.736\end{array}$} & \multirow{2}{*}{$\begin{array}{l}\text { Sig. } \\
.000^{\mathrm{b}}\end{array}$} \\
\hline & Regression & & & & & \\
\hline \multirow[t]{3}{*}{1} & Residual & 1080.985 & 76 & 14.223 & & \\
\hline & Total & 1845.295 & 77 & & & \\
\hline & Regression & 1136.107 & 2 & 568.054 & 60.074 & $.000^{\mathrm{c}}$ \\
\hline \multirow[t]{3}{*}{2} & Residual & 709.188 & 75 & 9.456 & & \\
\hline & Total & 1845.295 & 77 & & & \\
\hline & Regression & 1386.796 & 3 & 462.265 & 74.608 & $.000^{\mathrm{d}}$ \\
\hline \multirow[t]{3}{*}{3} & Residual & 458.498 & 74 & 6.196 & & \\
\hline & Total & 1845.295 & 77 & & & \\
\hline & Regression & 1587.603 & 4 & 396.901 & 112.436 & $.000^{\mathrm{e}}$ \\
\hline \multirow[t]{3}{*}{4} & Residual & 257.692 & 73 & 3.530 & & \\
\hline & Total & 1845.295 & 77 & & & \\
\hline & Regression & 1670.974 & 5 & 334.195 & 138.033 & $.000^{\mathrm{f}}$ \\
\hline \multirow[t]{3}{*}{5} & Residual & 174.321 & 72 & 2.421 & & \\
\hline & Total & 1845.295 & 77 & & & \\
\hline & Regression & 1717.294 & 6 & 286.216 & 158.759 & $.000^{\mathrm{g}}$ \\
\hline \multicolumn{7}{|c|}{ 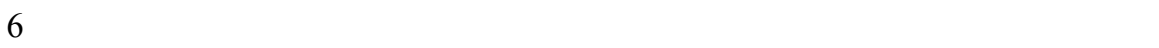 } \\
\hline & Residual & 128.001 & 71 & 1.803 & & \\
\hline
\end{tabular}




\begin{tabular}{|c|c|c|c|c|c|c|}
\hline & Total & 1845.295 & 77 & & & \\
\hline \multirow{3}{*}{7} & Regression & 1770.031 & 7 & 252.862 & 235.175 & $.000^{\mathrm{h}}$ \\
\hline & Residual & 75.264 & 70 & 1.075 & & \\
\hline & Total & 1845.295 & 77 & & & \\
\hline \multirow{3}{*}{8} & Regression & 1803.866 & 8 & 225.483 & 375.541 & $.000^{\mathrm{i}}$ \\
\hline & Residual & 41.429 & 69 & .600 & & \\
\hline & Total & 1845.295 & 77 & & & \\
\hline \multirow{3}{*}{9} & Regression & 1825.706 & 9 & 202.856 & 704.202 & $.000^{\mathrm{j}}$ \\
\hline & Residual & 19.588 & 68 & .288 & & \\
\hline & Total & 1845.295 & 77 & & & \\
\hline \multirow{3}{*}{10} & Regression & 1845.295 & 10 & 184.529 & . &.${ }^{\mathrm{k}}$ \\
\hline & Residual & .000 & 67 & .000 & & \\
\hline & Total & 1845.295 & 77 & & & \\
\hline
\end{tabular}

a. Dependent Variable: wempo;

b. Predictors: (Constant), Womentraining;

c. Predictors: (Constant), Womentraining, BroughtHarmonytohomes;

d. Predictors: (Constant), Womentraining, BroughtHarmonytohomes, Wmodernfarming;

e. Predictors: (Constant), Womentraining, BroughtHarmonytohomes, Wmodernfarming, Buyland;

f. Predictors: (Constant), Womentraining, BroughtHarmonytohomes, Wmodernfarming, Buyland, Finindependence;

g. Predictors: (Constant), Womentraining, BroughtHarmonytohomes, Wmodernfarming, Buyland, Finindependence, Expandbusiness;

h. Predictors: (Constant), Womentraining, BroughtHarmonytohomes, Wmodernfarming, Buyland, Finindependence, Expandbusiness, ContributehomeBudgets;

i. Predictors: (Constant), Womentraining, BroughtHarmonytohomes, Wmodernfarming, Buyland, Finindependence, Expandbusiness, ContributehomeBudgets, Loansaccessible;

j. Predictors: (Constant), Womentraining, BroughtHarmonytohomes, Wmodernfarming, Buyland, Finindependence, Expandbusiness, ContributehomeBudgets, Loansaccessible, wbuildhouses;

k. Predictors: (Constant), Womentraining, BroughtHarmonytohomes, Wmodernfarming, Buyland, Finindependence, Expandbusiness, ContributehomeBudgets, Loansaccessible, wbuildhouses, startbusiness. 


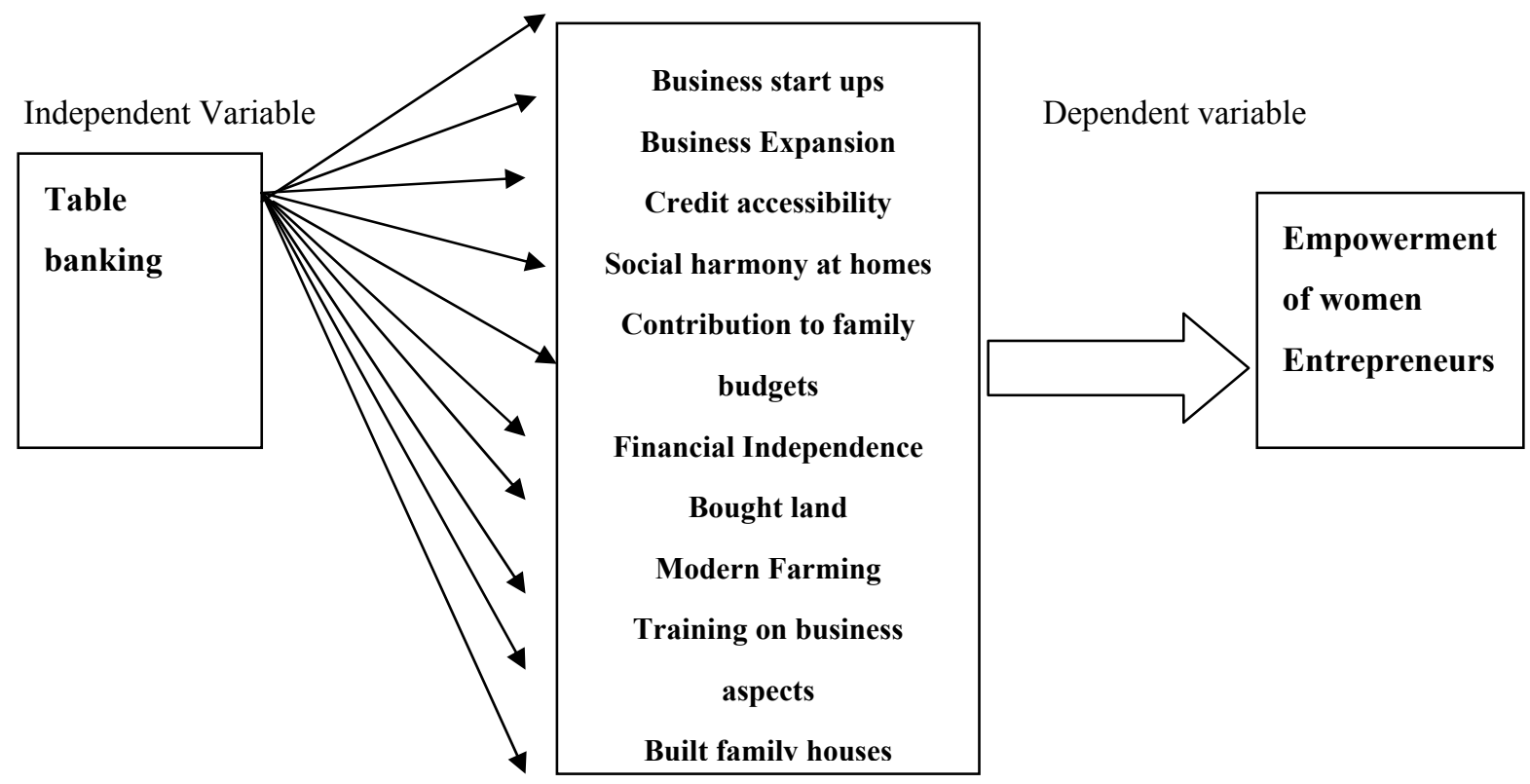

Figure 1. Framework of the Contributions of Table Banking to Empowerment of Women

\section{Entrepreneurs}

Source: Author, 2015.

\section{Discussion}

Table 1 shows that women belonging to Table banking age range from 5\% are $10-19,41 \%$ are $20-29$, $31 \%$ range between $30-39$ and $23 \%$ for 40 years and above. Youth seems to have embraced the Table banking concept because they form the bigger percent from the sample used. This could be because of various reasons such as unemployment and therefore trying to get economic empowerment, this could be true because by year 2011 unemployment rate in Kenya was 40 percent (Trading economics, 2012). This is in agreement with (Mureithi, 2014) who stated that, the major objective of Table banking is to give women economic empowerment through financial assistance and trainings. Group 3 ranging from 30-39 years took 23\%, this is shown even the older group are involved in Table banking. This is in agreement with Stewart (2014) who argues that today people enter into entrepreneurship out of need because they have been forced out of the corporate world. Many middle aged people are in the streets and turn to business to make a living and could be utilizing Table banking as a source of finance.

Table 2 depicts the marital status of the respondents $36 \%$ are married, $60 \%$ are single and $4 \%$ are divorced, implying that the single women could be engaging in this activity to support themselves and their dependents. A majority $60 \%$ of the respondents are single women who could be looking for support from the group because they have no spouses to turn to, they could be single parents, are 
unemployed among others, agreeing with (Thabarge, 2015) that being single is exhausting and therefore such people could be joining Table banking to get support.

As shown in (Table 3), the education level of the women in Table banking indicate $56 \%$ had tertiary level education, $26 \%$ secondary level education, while primary level education took about $13 \%$. The tertiary level respondents took lead because they could be more exposed, have financial knowledge than the secondary and primary level counterparts. Going through higher education changes people's mindset and this lead them utilize Table banking services to empower themselves financially. This could also have resulted due to unemployment level in Kenya.

As pertains the industry the respondents are operating from Table 4 shows that Hair \& Beauty and Finance took lead ranging 33\% and 28\%. Hair \& Beauty has become a booming business in developing countries, Kenya inclusive, this could be because of the existence of International brands such as Estee Lauder (USA), Oviflame (Sweden) and L'oreal (France), a growing number of middle class, a large growing consumer awareness and knowledge of personel grooming and urban migration (Euromonitor International, 2015). Today hair industry is considered an important industry opposite of what it was 20 years back (Kaloki, 2014), this could have attracted many entrepreneurs to join the industry and make their mark. Training in the beauty industry has also mushroomed hence presence of trained beauty personnel. Finance Industry is also growing this could be due to mushrooming of microfinance to serve the poor that have been cut off by the mainstream banks (Kota, 2007) hence a bigger percentage.

For experience (Table 5) depicts that those with least experience take lead in Table banking recording about $28 \%$, indicating that Table banking is being used as a source of economic empowerment more so for those without another source of finance. The most experienced entrepreneurs (above 5 years) according to the survey record $13 \%$, this could be due to the fact that their businesses are established and therefore are able to stand on their own or can afford to raise enough collateral to acquire a larger loan from the main stream banks. "As a custom before any financial institution lends out money, it checks if the person borrowing has the ability to return it' Kilongi (2011). This is opposite of Table banking that focuses on the potential of the person and not the material possession”.

Concerning the contribution made by Table banking towards empowering women entrepreneurs in Eldoret, Table 6 displays a normal distribution of variables by use of skewness and kurtosis which ranges from around 1-3. This is a positive indication. The mean though not very strong displays a great significance. Ranging from the strongest Table banking has contributed to food security, harmony in 
homes, enabled women to engage in modern farming, raised women standards of living, enabled women to build houses, given women ability to contribute to the family budgets, brought financial independence to women, enabled women to buy land, enabled accessibility of loans by women among others. All this truly empowers women, and when women are empowered they can do a variety of things such as feeding their family, starting a business or expanding an existing one. This is in agreement with Johncraig (2012) who adds that Table banking has led to the start of many income generating projects such as such as green houses, rearing of milk goats among others which enables them to get investment returns of over 200 percent. Table banking has led women to own land which customarily was only owned by men (Kilongi, 2011). This is a great achievement though it comes with a cost in terms of labour, logistics among others. For correlation analysis (Table 9) indicates a strong relationship between dependent variable and independent variables. This includes Table banking and business expansion. $412 *$ is significant at 0.5 , ability to buy land is at $.246^{*}$ at .05 , women training is at. $429 * *$ at .01 , starting of horticulture. $420 * *$ at .01 , loans accessibility is at $.443 * *$ at .01 among others. To support the model developed by the researcher (Figure 1), Table 10 the model summary depicts that women training through Table banking accounts to 40.6 percent variations in the empowered women metric. Table banking also led to harmony in homes, contributed to modern farming, buying of land, financial independence, start or expansion of businesses, contribution to home budgets, loan accessibility and building of houses among others are critical drivers of the level which women are empowered in Eldoret, Kenya. The Durbin-Watson value of 1.778 also resonates with the contribution that the determinates contribute to the level at which women are empowered in Eldoret, Kenya.

The univariate analysis of variance (Table 11) also established congruence or provided parallel support to the findings explicated by stepwise regression alluded to above. The F values and significant levels confirms the relationship among predictors and the dependable variables.

As regards the challenges facing women entrepreneurs as far as Table banking is concerned (Table 7) shows default cases took the lead recording a mean of about 2.5 , women could fail to pay their loans due to multiple financial responsibilities that they have, however this imparts badly on the group members and can cause disagreements. Record keeping also came strongly (mean 2.4) as a challenge, this could be because of lack of accounting background hence inability to reconcile properly. Other challenges recorded though they did not feature strongly were disagreements resulting due to non payments, non attendance of monthly meetings and failure to pay the monthly interest. There is also an 
indication of normal distribution of variables through skewness which ranges from .697-1.411. This shown positive indication.

Women also need to be receive financial management training in order to manage their finances effectively. This is an agreement with (Kumar, 2010) who states the importance of financial management is to offer a business protection from precarious mis-management of funds, enable it to pay for utilities, advise it to keep costs as low as possible and enable businesses to plan for tax payments. This could be of great help to women entrepreneurs who in most cases find themselves in the crossroads of family and business responsibilities.

Another strategy brought forward is restricting the number of Table banking groups a woman can join for efficiency purposes, this is also meant to create commitment and to retain the ability. Another strategy put forward (Table 8) is the introduction of collateral in the Table banking to minimize default cases. This agrees with Pritchard (2015) who state that collateral protects the lender from high risk. Collateral acts as a second chance of loan repayment and prevents the borrower from default which has harsh effects on the borrower's business and the personal life (Markowitz, 2011).

\section{Conclusion}

From the findings of the survey the following has been established: The biggest population comprising the Table banking groups in Eldoret, is the youth with 41 percent who are mostly single women, majority of the women in Table banking have attained tertiary education level, Hair and Beauty is the leading Industry for the women in the Table banking groups. Table banking has played a significant role in empowering women entrepreneurs in Eldoret: It has empowered them to buy land, start and expand their businesses, brought harmony at homes, given them financial independence, given them access to credits among others. Challenges facing Table banking in Eldoret are: Default cases, record keeping problems, disagreements for non payments, failure to attend meetings and failure to pay interests on the loans.

\section{Recommendations}

Based on the research findings, the following was recommended:

- Provision of financial and time management trainings to women;

- Introduction of a law that restricts the number of Table banking groups one can join;

- Introduction of collateral to edge default cases; 
- Involvement of Non Governmental Organizations in training women in different aspects of business aspects;

- $\quad$ Legalise Table banking to enable legal actions to be taken against defaulters;

- Lowering of monthly subscription to enable all women to manage the payments;

- $\quad$ More Table banking awareness and enlightenment programmes;

- $\quad$ Strict rules and regulations should be applied to all Table banking groups;

- $\quad$ Reduction of lending interest to minimize default cases and have timely payments;

- Men should also be encouraged to join to have more funds in the pool and to generate non bias ideas.

\section{References}

Abigael, S. (2014). President Uhuru encourages women on Table banking. Retrieved from http://www.standardmedia.co.ke

African Business Forum. (2014). Cape Town. Republic of South Africa.

Afroze. T., Rahman, S. M. T., \& Youuf, S. (2014). Multiple Borrowing through Microcredit and Its Impact on Loan Repayment: Study in Bangladesh. Research Journal in Accounting, 5(21).

Amunya, J. (2014). Women use locally available materials to start a beauty Industry in Lugari.

Asetto, F. (2014). Effect of Table banking on the investment decisions of Small \& Medium Enterprises in Nairobi County (Unpublished MBA project). University of Nairobi.

Bethwel, K. (2013). Joyful Women with a Midas Touch. The STAR. Retrieved from http://www.allafrica.com/stories/201312030423.html

Bula, H. O. (2012). Performance of Women in Small Scale Enterprises (SSEs) Marital Status and Family Characteristics. European Journal of Business and Management, 4(7).

Burns, R. A., \& Burns, R. B. (2008). Business Research Methods and Statistics Using SPSS: SAGE publications. Inc: London.

Gitau, R. M. (2011). The Relationship between financial innovation and financial performance on commercial banks in Kenya (Unpublised Thesis Nairobi).

Gray, E. D. (2009). Doing Research in the Real World (2nd ed.). Sage Publications Inc. London.

http:// www.standardmedia.co.ke

http://www. action.actionaid.org

http://www. joywo.org/about-us

http://www.uwezo.go.ke

ILO. (2001). Research Publication on: Growth Oriented Women Entrepreneuers in Kenya. Women Entrepreneues and Growth. World Bank Report.

John, C. D. (2012). Eliminate poverty Now. Retrieved from http://www.foodtank.com

Jonathan, A. (2013). No Slums in Nairobi. Retrieved from http://www.fieldnotesmagazine.depree.org/no-slums-in-nairobi

Kariithi, G. M. (2013). Challenges Affecting Accessibility of Credit Facilities Among Women Owned Enterprises in Nairobi Central Business District in Kenya JKUAT (Masters Thesis).

Kariuki, C. M., \& Ngugi, P. K. (2014). The effects of Table banking On The Performance Of Micro and Small Enterprises in Nairobi County. International Journal of Current Business and Social Sciences, 1(2), 339-360.

Kenya, C. (2009). Minister of State for Planning, National Development and Vision 2030. 
KenyaNews247.com. (2013). Women urged to form groups to enhance Table banking.

Kilongi, M. (2011). Chama the best choice for tjommies. Mail \& Gardian, Africa's Best read. Nairobi. Retrieved from http:/www.mg.co.za/article/2011-03-22-chama-the-best-choice-for-tjommies

Kimtai, A. (2015). Table-banking bridges the financial inclusion gap in Kenya. Standardmedia.co.ke.

Lumwamu, K., \& Murgor, B. (2014). How Joyful is changing the lives of rural women.

Mengo, B. (2014). Table-Banking, a booming loan facility among Kenyan women. English.news.cn. Nairobi.

Mungai, E. N., \& Ogot, M. (2012). Gender, Culture and Entrepreneurship in Kenya. International Business Research, 5(5). http://dx.doi.org/10.5539/ibr.v5n5p175

Municipal Council Of Eldoret. (2011). Government Printers.

Murithi, M. (2014). Ms. Ruto urges Kajuju to partner with Munya. Eastern Post. Retrieved from https:/www.theeasternpost.wordpress.com/2014/02/16

Njuguna, M. (2015). Table banking. The concept of Table banking.

Nyaga, B. (2013). Avoid Ethnicity and Embrace Table banking, Rachel Ruto to women.

Obiria, M. (2014). Table banking leads Kenya's women the means to beat the poverty trap. Retrieved from http:/www.theguardian.com/global-development/2014

Obiria, M. (2014). Table banking opens group's doors. DailyNation. Retrieved from http://www.nation.co.ke

Obiria, M. (2015). Kenyan Women Embrace Table banking to Break out of Poverty. Retrieved from http://www.thewip.net/2015/02/02

Ochieng, G. (2011). Women Group Spearheads poverty Battle. The African executive.

Omollo, K. (2015). Siaya woman killed over Sh 1,500 chama loan wragles. Standard Digital. Retrieved from http://www.standardmedia.co.ke

Quail, L. F. (2014). kenya: Mothers and Children scratching a living in Eldoret dump. The guardian. Retrieved from http://www.theguardian.com/global-development/2014

Rachel's Network. (2015). Sustainable Agriculture Depends On Women. Retrieved from http://www.rachelsnetwork.Org/root capital

Startup Academy. (2014). Chamas and Table banking A Viable Source of Funding. Retrieved from http://www.startupacademy.co.ke

Titus, T. (2013). Rachel Ruto roots for rural women Empowerment. Standard Digital. Retrieved from http://www.standardmedia.co.ke/

Tubey, R. J. (2012). NGO Intervention Measures, Performance and Trends of Women-Operated MSEs in Eldoret Municipality, Kenya. Journal of Emerging Trends in Economics and Management Sciences.

Tubey, R. J. (2013). The Influence of Socio-economic Characteristics of Women Entrepreneurs on the performance of their Micro Enterprise: The case of Eldoret Municipality in Uasin - Gishu County, Kenya. Herald International Research Journal, 2(1), 41-46.

Wambugu, B. (2016). Safaricom named in M-Kesho suit. Business Daily. Retrieved from http://www.businessdailyafrica.com

Women's Empowerment Principles. Equality MEans BusinEss. (2011). A partnership initiative of UN women and UN Global Compact.

Zita, M. A. (2013). How to borrow upto 9 times your chama savings. Chamasoft.

Zita, M. A. (2013). Successful Chamas needs rules. Chamasoft. 